\title{
EFFICIENCY OF MAIZE CULTIVARS FOR ZINC UPTAKE AND USE
}

\author{
Ângela Maria Cangiani Furlani ${ }^{1 *}$; Pedro Roberto Furlani ${ }^{1}$; Anderson Rotter Meda ${ }^{1}$; Aildson \\ Pereira Duarte ${ }^{2}$ \\ ${ }^{1}$ IAC - Centro de Pesquisa e Desenvolvimento de Solos e Recursos Ambientais, C.P. 28 - 13001-970 - Campinas, SP - \\ Brasil. \\ ${ }^{2}$ Polo Regional de Desenvolvimento Tecnológico dos Agronegócios do Médio Paranapanema (APTA), C.P. 263 - \\ 19800-000 - Assis, SP - Brasil. \\ *Corresponding author <afurlani@iac.sp.gov.br>
}

\begin{abstract}
Zinc deficiency usually occurs in maize grown in Brazilian acidic soils. The aim of this study was to evaluate commercial maize cultivars for their $\mathrm{Zn}$ uptake and utilization efficiency. A greenhouse experiment using nutrient solution with young plants was carried out at Campinas, State of São Paulo, Brazil, in 2002. Treatments consisted of: $0.0 ; 0.1 ; 0.2 ; 0.4$ and $0.8 \mathrm{mg} \mathrm{L}^{-1} \mathrm{Zn}$ in the main plots and 24 commercial maize cultivars in the subplots, in a randomized complete block design. Zn concentration in shoot dry matter (SDM) ranged from 28.4 to $41.6 \mathrm{mg} \mathrm{kg}^{-1}$ among cultivars, clearly indicating a dilution effect, since a negative relation between SDM and plant height was shown. Total $\mathrm{Zn}$-shoot content was a good parameter to discriminate cultivars, once correlated with plant height and SDM $\left(r=0.66^{* *}\right.$ and $r=0.67^{* *}$, respectively). Analysis of variance and polynomial regression for total $\mathrm{Zn}$-shoot content was highly significant among cultivars and for the interaction cultivar vs $\mathrm{Zn}$-concentration. Plants under low $\mathrm{Zn}$ presented up to three-fold differences among efficiency index values (E.I. $=8.59$ to $26.42 \mathrm{mg}^{2} \mathrm{DM} \mu \mathrm{g}^{-1} \mathrm{Zn}$ ). The results with young plants indicated six maize cultivars classified as Zn-efficient and responsive (AG 7575, Tork, AL Bandeirante, AL 34, AGN 2012, Master) and six cultivars classified as efficient non-responsive (P30F33, P30K75, P30F80, AS 1533, DOW 8420 e AL 30). Other nutrient concentrations in the SDM were within normal limits $(\mathrm{K}, \mathrm{P}, \mathrm{Ca}, \mathrm{Mg}, \mathrm{Cu}, \mathrm{Fe}, \mathrm{Mn}$ ) for maize young plants.

Key words: Zea mays L., Zn-efficiency, Zn uptake, Zn-deficiency, commercial cultivars
\end{abstract}

\section{EFICIÊNCIA DE CULTIVARES DE MILHO NA ABSORÇÃO E UTILIZAÇÃO DE ZINCO}

\begin{abstract}
RESUMO: Nos solos ácidos são comuns os casos de deficiência de zinco ( $\mathrm{Zn}$ ) na cultura do milho. O objetivo do presente trabalho consistiu em avaliar cultivares comerciais de milho quanto à eficiência na absorção e utilização de Zn. O experimento foi conduzido em Campinas, SP, Brasil, 2002, em casa de vegetação com plantas jovens em solução nutritiva, utilizando blocos ao acaso em parcelas divididas, sendo os tratamentos: 0,$0 ; 0,1 ; 0,2 ; 0,4$ e $0,8 \mathrm{mg} \mathrm{L}^{-1}$ de $\mathrm{Zn}$ e 24 cultivares comerciais de milho. Os teores de $\mathrm{Zn}$ na parte aérea (PA) variaram de 28,4 a $41,6 \mathrm{mg} \mathrm{kg}^{-1}(1,46 \mathrm{vez})$ entre as cultivares. O conteúdo total de $\mathrm{Zn}$ na PA foi o parâmetro que melhor se correlacionou com a altura de planta $\left(\mathrm{r}=0,66^{* *}\right)$ e com a matéria seca de parte aérea (MSPA) $\left(\mathrm{r}=0,67^{* *}\right)$, permitindo diferenciação das cultivares. A análise da variância e a regressão polinomial para essa variável revelaram diferenças significativas entre cultivares, bem como para o índice de eficiência, cujos valores variaram de até três vezes $\left(8,59\right.$ a $\left.26,42 \mathrm{mg}^{2} \mathrm{MS} \mu \mathrm{g}^{-1} \mathrm{Zn}\right)$ em condições de baixo $\mathrm{Zn}$. Os resultados com plantas jovens indicaram seis cultivares como eficientes e responsivas (AG 7575, Tork, AL Bandeirante, AL 34, AGN 2012, Master) e outras seis como eficientes e não-responsivas (P30F33, P30K75, P30F80, AS 1533, DOW 8420 e AL 30). Os teores de K, P, Ca, Mg, Cu, Fe e Mn na MSPA, estiveram dentro dos limites normais para plântulas de milho.

Palavras-chave: Zea mays L., absorção de Zn, deficiência de Zn, cultivares comerciais
\end{abstract}

\section{INTRODUCTION}

Acidic low fertility and/or lime-amended high fertility soils frequently present micronutrient deficiencies in annual crops and perennial plantations, specially $\mathrm{Zn}$ deficiency in maize, coffee and citrus, which are considered to be highly responsive to $\mathrm{Zn}$ fertilization.
Plant demands for $\mathrm{Zn}$ vary among species and cultivars. Differential cultivar responses grown under low soil $\mathrm{Zn}$ concentrations have been reported in maize, millet, sorghum, rice and wheat, among others (Brown et al., 1972; Clark, 1978; Safaya \& Gupta, 1979; Shukla \& Raj, 1987; Cakmak et al., 1999; Fageria, 2001). In a study comparing species, Zn uptake efficiency was evaluated 
in potato, wheat, maize and sunflower assessed by shoot dry matter yields (Trehan \& Sharma, 2000), and sunflower plants were found to be the most $\mathrm{Zn}$-efficient.

Root morphology and physiology have been related to genotype ability to overcome $\mathrm{Zn}$ deficiency, interfering on the processes of zinc acquisition and release of exudates in the rizosphere (Cakmak et al., 1998; Erenoglu et al., 1999); and also, on differential Zn transport along the vascular system, resulting in variations in $\mathrm{Zn}$ distribution and remobilization to vegetative parts and filling grains (Pearson \& Rengel, 1995a; 1995b; Pearson et al., 1996a; 1996b; Pearson et al., 1998; 1999).

Maize responses to low $\mathrm{Zn}$ in soil and nutrient solution have been evaluated through variations in dry matter yields, tissue mineral composition (Safaya \& Gupta, 1979). These authors obtained a significant reduction in total plant dry matter production, due to $\mathrm{Zn}$ deficiency, varying from $26.6 \%$ to $74 \%$, depending on the cultivar. Kuz-Menko et al. (1994) studied nine maize inbred lines and hybrids for differential Zn uptake and tissue concentration in 14-day-old plants and obtained a high relationship between $\mathrm{Zn}$ tissue accumulation at early plant stages and stimulation on ontogeny and dry matter yield. Genotype dry matter yield variation of about $10 \%$ was observed and related to tryptophan synthase activity in function of $\mathrm{Zn}$ supply to young plants.

Hopkins et al. (1998) compared the quantity of phytosiderophores in the rizosphere of wheat, sorghum and maize under $\mathrm{Zn}$ deficiency in nutrient solution and found out that wheat and sorghum roots released higher quantity of exudates as compared to maize. This fact together with the higher maize $\mathrm{Zn}$ demand for growth might explain the causes of the prevalence of $\mathrm{Zn}$ deficiency in field grown maize as compared to the two other species under the same conditions.

The investigation on germplasm variation for $\mathrm{Zn}$ efficiency of several species has stimulated the establishment of plant breeding programs aiming at the selection of plants for this character, once this metal is a highly relevant nutrient for crop yield improvement and also for human nutrition (Cakmak et al., 1999).

The objective of this research was to evaluate commercial maize cultivars for $\mathrm{Zn}$ uptake and utilization efficiency using young plants grown in several $\mathrm{Zn}$ concentrations.

\section{MATERIAL AND METHODS}

The experiment was carried out under greenhouse conditions, at Campinas, SP, Brazil. The experimental design consisted of randomized complete blocks, in splitplots with three replications. Treatments in the main plots were the $\mathrm{Zn}$ concentrations $(0.0 ; 0.1 ; 0.2 ; 0.4$ and $0.8 \mathrm{mg}$ $\mathrm{L}^{-1}$ ) and in the subplots, 24 maize cultivars currently available in the market.

Seeds were germinated in moistened paper (type Germitest) and seven-day-old seedlings were transplanted to recipients with nutrient solution. The experimental units consisted of 15 L-plastic recipients filled with nutrient solution and an acrylic lid on top with holes (silver painted to avoid light in roots) holding a total of 168 plants per recipient: seven seedlings of each cultivar, inserted in the holes and supported by sponge beads.

The basic nutrient solution consisted of (in $\mathrm{mg}$ $\left.\mathrm{L}^{-1}\right)$ : Ca 160: K 160; Mg 19.5; N $158\left(\mathrm{~N}^{-\mathrm{NO}_{3}} 138.0+\mathrm{N}-\right.$ $\left.\mathrm{NH}_{4} 20.0\right) ; \mathrm{S} 64 ; \mathrm{B}=33 ; \mathrm{Cl} 32.5 ; \mathrm{P} 10.0 ; \mathrm{Fe} 3.6 ; \mathrm{Mn}$ 0.65; $\mathrm{Cu} 0.05$ and Mo 0.08 (Furlani \& Furlani, 1988) (Table 1). The electrical conductivity (E.C.) was $1.51 \mathrm{mS}$ $\mathrm{cm}^{-1}$. Nutrient solution was kept under continuous aeration and the $15 \mathrm{~L}$ volume was maintained by daily additions of deonized water. The initial solution $\mathrm{pH}$ was adjusted to 5.0 (with $0.1 \mathrm{~mol} \mathrm{~L}^{-1} \mathrm{NaOH}$ ) and monitored during the experimental period. The solution E.C. was adjusted three times during the experiment by the addition of stock solutions: solution A (stocks $1+5+6$ ) and solution B (stocks $2+3+4$ ) in the same proportions described in Table 1, but 50 fold-concentrated. At 3, 8 and 15 days after transplanting $18.6 \mathrm{~mL}$ of each concentrated stock-

Table 1 - Composition of stock solutions used in preparation of the nutrient solution ${ }^{1}$.

\begin{tabular}{llcc}
\hline $\begin{array}{l}\text { Stock } \\
\text { solution }\end{array}$ & \multicolumn{1}{c}{ Salts p.a. } & $\begin{array}{c}\text { Concentration of } \\
\text { stock solution }\end{array}$ & $\begin{array}{c}\text { Proportion of stock in } \\
\text { nutrient solution }\end{array}$ \\
\hline & & $\mathrm{g} \mathrm{L}^{-1}$ & $\mathrm{~mL} \mathrm{~L}^{-1}$ \\
1 & $\mathrm{Ca}\left(\mathrm{NO}_{3}\right)_{2} \cdot 4 \mathrm{H}_{2} \mathrm{O} / \mathrm{NH}_{4} \mathrm{NO}_{3}$ & $270.0 / 33.8$ & 3.30 \\
2 & $\mathrm{KCl} / \mathrm{K}_{2} \mathrm{SO}_{4} / \mathrm{KNO}_{3}$ & $18.6 / 44.0 / 24.6$ & 3.60 \\
3 & $\mathrm{MgSO}_{4} \cdot 7 \mathrm{H}_{2} \mathrm{O}$ & 136.9 & 1.40 \\
4 & $\mathrm{KH}_{2} \mathrm{PO}_{4}$ & 35.1 & 8.00 \\
5 & $\mathrm{FeSO}_{4} \cdot 7 \mathrm{H}_{2} \mathrm{O} / \mathrm{HEDTA}_{2}$ & $9.16 / 8.68$ & 2.00 \\
6 & $\mathrm{MnCl}_{2} \cdot 4 \mathrm{H}_{2} \mathrm{O} / \mathrm{Na}_{2} \mathrm{MoO}_{4} \cdot 2 \mathrm{H}_{2} \mathrm{O} /$ & $2.34 / 0.26 / 0.20 / 2.04$ & 1.00 \\
7 & $\mathrm{CuSO}_{4} \cdot 5 \mathrm{H}_{2} / \mathrm{OH}_{3} \mathrm{BO}_{3}$ & 43.986 & $0.0-0.01-0.02-0.04$ and $0.08 \mathrm{~mL}$ \\
\hline
\end{tabular}

${ }^{1}$ Furlani \& Furlani (1988). 
solution A and B was added for each $1 \mathrm{~L}$ of nutrient solution and each $0.1 \mathrm{mS} \mathrm{cm}^{-1}$ lower than the original value (1.51), in order to maintain similar E.C. in all recipients.

The maximum and minimum air temperature and relative humidity means and mean standard deviations were, respectively: $31.4 \pm 2.5^{\circ} \mathrm{C}$ and $16.4 \pm 0.8^{\circ} \mathrm{C}$; and $61.7 \pm 11.8 \%$ and $14.1 \pm 5.2 \%$.

Plants were harvested after 17 days, placed in plastic bags and kept in a cooled room for plant height determination. Thereafter, plants were rinsed in distilled water, blotted dry, placed in paper bags and taken to a forced-air oven at $70^{\circ} \mathrm{C}$, until constant weight. After this, the following determinations were made: shoot dry matter yield and $\mathrm{Zn}, \mathrm{P}, \mathrm{Ca}, \mathrm{K}, \mathrm{Mg}, \mathrm{B}, \mathrm{Fe}, \mathrm{Cu}$ and $\mathrm{Mn}$ concentrations. Plant samples were oven digested at $450^{\circ} \mathrm{C}$ and analysed for $\mathrm{K}$ by flame-emission photometry; and for the other nutrients by ICP-OES spectrometry. Calculations of Zn-utilization efficiency index (E.I.) for plant shoot dry matter yield were made according to Siddiqi \& Glass (1981): $\mathrm{EI}=$ shoot DM / [shoot Zn-conc] = $(\text { Shoot DM })^{2} /[$ total $\mathrm{Zn}$ content $]=\mathrm{mg}^{2} \mathrm{DM} \cdot \mathrm{mg}^{-1} \mathrm{Zn}$.

Data was submitted to analysis of variance and polynomial regression for all variables. Mean comparisons made by the Duncan test $(0.05)$.

\section{RESULTS AND DISCUSSION}

No severe typical visual symptoms of $\mathrm{Zn}$ deficiency were observed in the control plants, although they showed a great reduction in shoot height. Zn-deficiency causes internode shortening and decreases leaf size. Chemical analysis of the nutrient solutions in the beggining of the experiment indicated precision for the treatments: $0.00,0.12,0.22,0.44$ and $0.86 \mathrm{mg} \mathrm{Zn} \mathrm{L}^{-1}$ (deonized water and pure salts were used in the preparation of nutrient solutions). Because of the absence of visual $\mathrm{Zn}$ symptoms, original seed samples were analyzed for $\mathrm{Zn}$ contents and other nutrients in order to verify whether seeds could have supplied seedlings with enough $\mathrm{Zn}$ to avoid the appearance of severe deficiency symptoms. A range of 13 to $91 \mu \mathrm{g} \mathrm{Zn} \mathrm{g}{ }^{-1}$ expressed in a whole seed dry matter basis was found (average of $25 \mu \mathrm{g} \mathrm{g}^{-1}$ ). Bityutskii et al. (1999; 2000; 2002) analyzed metal contents $(\mathrm{Ca}, \mathrm{Fe}, \mathrm{Mn}$ and $\mathrm{Zn}$ ) of several maize grain parts and found a positive correlation between these metal concentrations and root growth during germination. Mature grains of 25 maize genotypes were classified into groups according to the Fe, Mn and $\mathrm{Zn}$ contents in the scutellum. High and low-Zn genotypes were those containing 89-94 and 76

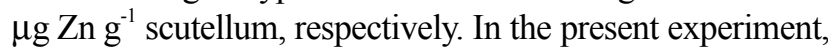
the seed $\mathrm{Zn}$ contents determined in the original seed material were expressed in a whole seed basis and the average $\mathrm{Zn}$ concentration $\left(25 \mu \mathrm{g} \mathrm{g}^{-1}\right)$ appeared to be sufficient for the initial seedling supply, since no typical visual symptoms of $\mathrm{Zn}$ deficiency were observed.
Dry matter yield varied, in average, 2.43 times among cultivars (from $238 \mathrm{mg}$ to $579 \mathrm{mg}$ per plant). The analysis of variance showed highly significant $(P<0.01)$ differences among cultivars and also a significant interaction among cultivars and $\mathrm{Zn}$ rates. The polynomial regression analysis for dry matter production in function of Zn concentrations was significant for cultivars $01,07,12$, $13,14,17$ and 20 , even though not significant for the others (Table 2; Figure 1).

Plant height varied 1.34-fold, in average, among cultivars (from 58.4 to $78.6 \mathrm{~cm}$ ). The analysis of variance and polynomial regression revealed highly significant differences among cultivars, as well for the interaction of cultivars and rates of $\mathrm{Zn}$. In fact, the polynomial regression (linear or quadratic) for plant height as a function of $\mathrm{Zn}$ was significant only for cultivars $01,12,13,16$, 17 and 20. Plant height was apparently a less discriminative parameter than shoot dry matter yield, in response to $\mathrm{Zn}$ rates (Table 3 ).

Once the interaction among cultivars and $\mathrm{Zn}$ concentrations was highly significant for dry matter production as well as for plant shoot height, it was necessary to look at each individual plant behavior and to determine the maximum point in the cases there was a quadratic type response. At the highest $\mathrm{Zn}$ rates, several cultivars presented reduction in growth, evidencing a higher susceptibility to the metal, while others showed a linear response (Tables 2 and 3). Plant responses in dry matter yield and height clearly indicate that the $\mathrm{Zn}$ demand varied among cultivars, and that the $\mathrm{Zn}$ concentration in nutrient solution and in plant dry tissue for maximal dry matter yield might be estimated. Since, in this experiment, significant $F$ values for linear or quadratic polinomial regression were obtained for some cultivars (Table 2) individual quadratic equations were found for the cultivars $01,07,13$, 17 and 20, resulting in the following $\mathrm{Zn}$ concentrations

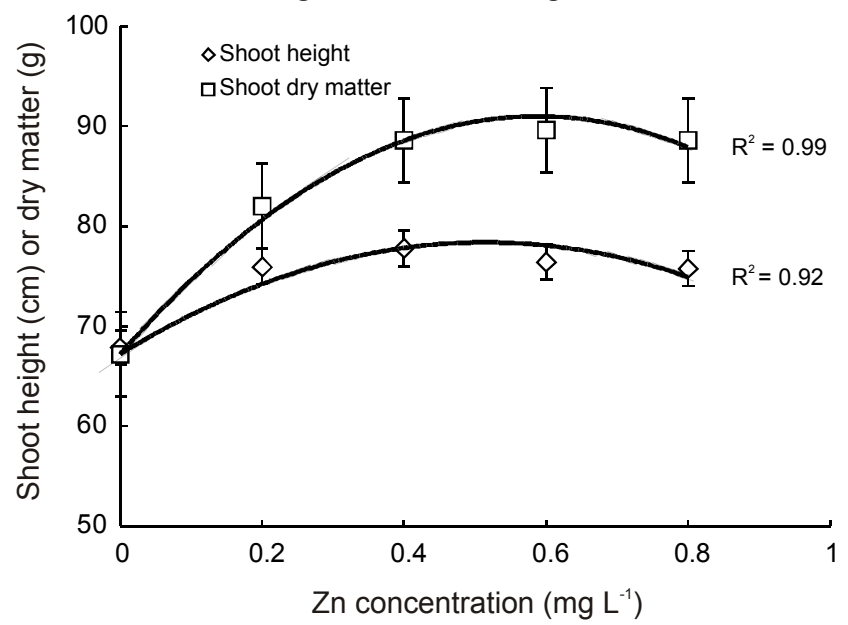

Figure 1 - Shoot height $(\mathrm{cm})$ and shoot dry matter yield (relative scale: $\mathrm{g} / 200$ plants) in maize plants grown under increasing $\mathrm{Zn}$ concentrations. (Means of 24 cultivars, 72 observations). 
Table 2 - Shoot dry matter of 24 day-old maize plants grown under increasing Zn concentrations.

\begin{tabular}{|c|c|c|c|c|c|c|}
\hline \multirow{2}{*}{$\begin{array}{l}\text { Maize } \\
\text { cultivar }\end{array}$} & \multicolumn{6}{|c|}{$\mathrm{Zn}$ concentrations $\left(\mathrm{mg} \mathrm{L}^{-1}\right)$} \\
\hline & 0.0 & 0.1 & 0.2 & 0.4 & 0.8 & Mean \\
\hline & 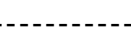 & 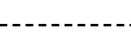 & $\ldots-\ldots m$ & $-1-\ldots-1$ & $--1-1$ & $-\cdots$ \\
\hline 10- AL 34 (Var) & $438 \mathrm{ab}$ & $727 \mathrm{a}$ & $606 \mathrm{ab}$ & 542 bcd & 582 a-e & 579 \\
\hline 07- AS $1533\left(\mathrm{MSH}^{2}\right)$ & $458 \mathrm{a}$ & $543 \mathrm{~b}$ & $638 \mathrm{a}$ & $619 a b$ & 483 a-h & 548 \\
\hline 06 - Tork (SH) & 387 a-d & $547 \mathrm{~b}$ & $574 \mathrm{abc}$ & $521 \mathrm{~b}-\mathrm{e}$ & $623 a b c$ & 530 \\
\hline 17- AGN $2012(\mathrm{DH})$ & 383 a-d & $485 \mathrm{bc}$ & 515 a-e & $586 \mathrm{abc}$ & $644 \mathrm{a}$ & 523 \\
\hline 20- Master $(\mathrm{TH})$ & 373 a-e & $532 \mathrm{~b}$ & 485 a-f & 532 bcd & 618 a-d & 508 \\
\hline 04- P30F33 (SH) & $449 a b$ & $502 \mathrm{~b}$ & 566 a-d & $453 \mathrm{~b}-\mathrm{h}$ & $452 \mathrm{c}-\mathrm{i}$ & 484 \\
\hline 03- P30K75 (SH) & $411 \mathrm{a}-\mathrm{d}$ & $430 \mathrm{bcd}$ & 562 a-d & $508 \mathrm{~b}-\mathrm{f}$ & $468 \mathrm{~b}-\mathrm{i}$ & 476 \\
\hline 09- ALBandeirante $\left(\operatorname{Var}^{3}\right)$ & 363 a-e & $481 \mathrm{bc}$ & 568 a-d & $493 \mathrm{~b}-\mathrm{g}$ & 476 a-h & 476 \\
\hline 13- AG $122(\mathrm{DH})$ & $276 \mathrm{~b}-\mathrm{e}$ & 442 bcd & $447 \mathrm{~b}-\mathrm{g}$ & 729 a & 490 a-g & 476 \\
\hline $01-\mathrm{AG} 7575\left(\mathrm{SH}^{1}\right)$ & 386 a-d & $478 \mathrm{bc}$ & $449 \mathrm{~b}-\mathrm{g}$ & $579 \mathrm{abc}$ & $436 \mathrm{e}-\mathrm{i}$ & 466 \\
\hline 12- AG $1051\left(\mathrm{DH}^{4}\right)$ & 304 a-e & $399 \mathrm{~b}-\mathrm{e}$ & $553 \mathrm{a}-\mathrm{d}$ & $413 c-j$ & $628 \mathrm{ab}$ & 459 \\
\hline 11- AL 30 (Var) & 378 a-d & $450 \mathrm{bcd}$ & 516 a-e & $353 e-j$ & $449 \mathrm{~d}-\mathrm{i}$ & 429 \\
\hline 02- P30F80 (SH) & $419 \mathrm{abc}$ & $416 \mathrm{~b}-\mathrm{e}$ & $430 \mathrm{~b}-\mathrm{g}$ & $488 \mathrm{~b}-\mathrm{h}$ & $377 \mathrm{f}-\mathrm{j}$ & 426 \\
\hline 08- DOW 8420c (MSH) & 360 a-e & $417 \mathrm{~b}-\mathrm{e}$ & 482 a-f & $438 \mathrm{c}-\mathrm{i}$ & $399 \mathrm{f}-\mathrm{j}$ & 419 \\
\hline 14- Traktor $(\mathrm{DH})$ & $269 \mathrm{~b}-\mathrm{e}$ & $380 \mathrm{~b}-\mathrm{f}$ & $354 \mathrm{e}-\mathrm{i}$ & $437 \mathrm{c}-\mathrm{i}$ & 510 a-f & 390 \\
\hline 05- AS $1545(\mathrm{SH})$ & 298 a-e & $373 b-f$ & 394 d-h & $373 \mathrm{~d}-\mathrm{j}$ & $391 \mathrm{f}-\mathrm{j}$ & 365 \\
\hline 15- BRS $2223(\mathrm{DH})$ & 310 a-e & $398 \mathrm{~b}-\mathrm{e}$ & $352 \mathrm{e}-\mathrm{i}$ & $382 \mathrm{~d}-\mathrm{j}$ & $364 \mathrm{f}-\mathrm{j}$ & 361 \\
\hline 21- BRS $3060(\mathrm{TH})$ & 312 a-e & $296 \mathrm{~d}-\mathrm{g}$ & $318 \mathrm{f}-\mathrm{i}$ & $333 \mathrm{f}-\mathrm{j}$ & $379 \mathrm{f}-\mathrm{j}$ & 328 \\
\hline 16- BRS $2114(\mathrm{DH})$ & 245 cde & $309 \mathrm{c}-\mathrm{g}$ & $330 \mathrm{f}-\mathrm{i}$ & $424 c-j$ & $317 \mathrm{~g}-\mathrm{j}$ & 325 \\
\hline 18- XB $8010(\mathrm{DH})$ & 243 cde & $324 \mathrm{c}-\mathrm{g}$ & $280 \mathrm{ghi}$ & $402 \mathrm{~d}-\mathrm{j}$ & $333 \mathrm{f}-\mathrm{j}$ & 316 \\
\hline 24- Exceler (TH) & $230 \mathrm{de}$ & $211 \mathrm{fg}$ & $415 \mathrm{c}-\mathrm{h}$ & $320 \mathrm{~g}-\mathrm{j}$ & $377 \mathrm{f}-\mathrm{j}$ & 311 \\
\hline 22- AG $6690(\mathrm{TH})$ & 321 a-e & $251 \mathrm{efg}$ & $328 \mathrm{f}-\mathrm{i}$ & 314 hij & 311 hij & 305 \\
\hline 19- AGN $3150\left(\mathrm{TH}^{5}\right)$ & $251 \mathrm{cde}$ & $245 \mathrm{efg}$ & $209 \mathrm{i}$ & $274 \mathrm{ij}$ & $237 \mathrm{j}$ & 243 \\
\hline 23- AGN $3180(\mathrm{TH})$ & $197 \mathrm{e}$ & $198 \mathrm{~g}$ & $253 \mathrm{hi}$ & $248 \mathrm{j}$ & $292 \mathrm{ij}$ & 238 \\
\hline Means & 336 & 410 & 443 & 448 & 443 & \\
\hline
\end{tabular}

${ }^{1} \mathrm{SH}=$ single cross hybrid; ${ }^{2} \mathrm{MSH}=$ modified $\mathrm{SH} ;{ }^{3} \mathrm{Var}=$ variety; ${ }^{4} \mathrm{DH}=$ double cross hybrid; ${ }^{5} \mathrm{TH}=$ three-way cross hybrid. Linear polynomial regression (L) and/or quadratic $(\mathrm{Q})$ were significant only for cultivars $01,07,12,13,14,17$ and 20 . Test $\mathrm{F}($ cultivar) $=16.7 * *$; $\mathrm{F}$ interaction $(\mathrm{Zn}$ vs cult $)=1.37^{*} ; \mathrm{CV} \%(\mathrm{Zn})=5.5 ; \mathrm{CV} \%(\mathrm{cult})=22.0$. Means followed by the same letters, in columns, do not differ by Duncan's test (0.05).

for maximal dry matter yields: cultivar $01(\mathrm{AG} 7575)-\mathrm{Zn}$ tissue concentration $=38 \mathrm{mg} \mathrm{kg}^{-1}$; maximal dry matter $=$ $523 \mathrm{mg}$ plant $^{-1}$; cultivar 07 (AS 1533) - 33 and 624; cultivar 13 (AG 122) - 37 and 628; cultivar 17 (AGN 2012) 47 and 648; and cultivar 20 (Master) - 46 and 616, respectively. These cultivars were more efficient at low $\mathrm{Zn}$ concentrations and more susceptible or less tolerant to high $\mathrm{Zn}$. The $\mathrm{Zn}$ concentrations for maximal shoot dry matter yield found for cultivars $01,07,13,17$ and 20 may be considered an approach for the critical level for deficiency or toxicity. They are very close to the results obtained by Perveen (2000), when comparing bioavailable $\mathrm{Zn}$ in soil and plant tissues, using the maize cultivar Azam as a test crop: a critical $\mathrm{Zn}$ level was found around $34-35 \mathrm{mg} \mathrm{kg}^{-1}$ by graphical and statistical methods.

Nevertheless, some other maize cultivars presented a linear response in shoot dry matter yield as $\mathrm{Zn}$ increased in the solution, indicating a higher demand for $\mathrm{Zn}$ and higher critical levels: 12 (AG 1051); 13 (AG 122);
14 (Traktor); 16 (BRS 2114); 18 (XB 8010); 23 (AGN 3180); 24 (Exceler), These cultivars were low Zn-efficient and more tolerant to higher $\mathrm{Zn}$ concentrations (Table 2).

Shoot- $\mathrm{Zn}$ concentrations varied from 28.4 to 41.6 $\mathrm{mg} \mathrm{kg}^{-1}$ (1.46-fold) among cultivars, but with inversely proportional values in relation to dry matter production and shoot height, indicating a dilution effect. Differences among cultivars were highly significant, but no significant interaction between cultivars and $\mathrm{Zn}$ rates was found; $\mathrm{Zn}$ concentrations in plant shoot increased as $\mathrm{Zn}$ increased in nutrient solution. Polynomial regression was highly significant for all cultivars (Tables 2, 3 and 4). Figure 2 illustrates the relationship for the 24 cultivar means, among shoot $\mathrm{Zn}$-concentrations and the external $\mathrm{Zn}$-concentrations, which was adjusted by a linear equation ( $R$ $\left.=0.94^{* *}\right)$. However, there was a low correlation between shoot-Zn concentrations and shoot dry matter yield $(\mathrm{r}=$ $-0.10^{*}$ ) and between shoot-Zn concentrations and shoot height $\left(r=0.10^{*}\right)$ (Table 7$)$. 
Table 3 - Shoot height of 24-day-old maize plants grown under increasing $\mathrm{Zn}$ concentrations.

\begin{tabular}{|c|c|c|c|c|c|c|}
\hline \multirow{2}{*}{$\begin{array}{l}\text { Maize } \\
\text { cultivar }\end{array}$} & \multicolumn{6}{|c|}{ Zn concentrations $\left(\mathrm{mg} \mathrm{L}^{-1}\right)$} \\
\hline & 0.0 & 0.1 & 0.2 & 0.4 & 0.8 & Mean \\
\hline 10- AL 34 (Var) & $65.5 \mathrm{a}-\mathrm{c}$ & $86.5 \mathrm{a}$ & 80.8 a & $79.3 \mathrm{a}$ & $81.0 \mathrm{a}-\mathrm{b}$ & 78.6 \\
\hline 08- DOW 8420c (MSH) & $70.7 \mathrm{a}$ & $76.2 \mathrm{~b}-\mathrm{d}$ & 77.6 a-c & $76.2 \mathrm{a}-\mathrm{c}$ & 77.4 a-e & 75.6 \\
\hline $07-\mathrm{AS} 1533\left(\mathrm{MSH}^{2}\right)$ & $65.1 \mathrm{a}-\mathrm{c}$ & $77.0 \mathrm{~b}$ & 76.8 a-c & $78.6 \mathrm{a}$ & 77.5 a-e & 75.5 \\
\hline 06 - Tork (SH) & $64.7 \mathrm{a}-\mathrm{c}$ & $76.4 \mathrm{~b}-\mathrm{d}$ & $77.4 \mathrm{a}-\mathrm{c}$ & $75.6 \mathrm{a}-\mathrm{c}$ & $82.1 \mathrm{a}$ & 75.2 \\
\hline $03-\mathrm{P} 30 \mathrm{~K} 75(\mathrm{SH})$ & $67.9 \mathrm{ab}$ & $75.9 \mathrm{~b}-\mathrm{d}$ & 77.8 a-c & $76.4 \mathrm{ab}$ & 75.8 a-e & 74.8 \\
\hline 09- ALBandeirante $\left(\operatorname{Var}^{3}\right)$ & $65.4 \mathrm{a}-\mathrm{c}$ & $75.7 \mathrm{~b}-\mathrm{d}$ & $79.1 \mathrm{a}-\mathrm{b}$ & 74.7 a-d & 78.7 a-e & 74.7 \\
\hline 17- AGN 2012 (DH) & $65.1 \mathrm{a}-\mathrm{c}$ & $74.6 \mathrm{~b}-\mathrm{d}$ & 72.9 a-d & 75.9 a-c & 80.8 a-c & 73.9 \\
\hline 20- Master (TH) & $64.7 \mathrm{a}-\mathrm{c}$ & $76.7 \mathrm{bc}$ & 73.8 a-d & $74.2 \mathrm{a}-\mathrm{d}$ & 79.9 a-d & 73.9 \\
\hline 04- P30F33 (SH) & $67.5 \mathrm{ab}$ & $76.3 \mathrm{~b}-\mathrm{d}$ & $77.4 \mathrm{a}-\mathrm{c}$ & 72.3 a-e & $74.3 \mathrm{a}-\mathrm{f}$ & 73.6 \\
\hline 02- P30F80 (SH) & $68.2 \mathrm{ab}$ & $74.7 \mathrm{~b}-\mathrm{d}$ & 73.9 a-d & $76.8 \mathrm{ab}$ & $73.7 \mathrm{~b}-\mathrm{f}$ & 73.4 \\
\hline $01-\mathrm{AG} 7575\left(\mathrm{SH}^{1}\right)$ & $60.5 \mathrm{~b}-\mathrm{d}$ & $74.9 \mathrm{~b}-\mathrm{d}$ & $72.8 \mathrm{~b}-\mathrm{d}$ & $76.7 \mathrm{ab}$ & $74.3 \mathrm{a}-\mathrm{f}$ & 71.9 \\
\hline 05- AS 1545 (SH) & 66.9 a-c & $73.3 \mathrm{~b}-\mathrm{d}$ & $72.6 \mathrm{~b}-\mathrm{d}$ & $71.6 \mathrm{a}-\mathrm{e}$ & $73.8 \mathrm{~b}-\mathrm{f}$ & 71.6 \\
\hline 13- AG $122(\mathrm{DH})$ & 52.5 ef & $74.2 \mathrm{~b}-\mathrm{d}$ & $72.0 \mathrm{~b}-\mathrm{e}$ & $77.6 \mathrm{ab}$ & 75.7 a-e & 70.4 \\
\hline 11- AL 30 (Var) & $64.1 \mathrm{a}-\mathrm{c}$ & $73.6 \mathrm{~b}-\mathrm{d}$ & 74.5 a-d & $66.5 \mathrm{e}-\mathrm{g}$ & $73.0 \mathrm{c}-\mathrm{f}$ & 70.3 \\
\hline 18- XB $8010(\mathrm{DH})$ & $61.5 \mathrm{~b}-\mathrm{d}$ & $72.6 \mathrm{~b}-\mathrm{e}$ & $67.4 \mathrm{~d}-\mathrm{g}$ & $75.4 \mathrm{a}-\mathrm{c}$ & $72.0 \mathrm{~d}-\mathrm{f}$ & 69.8 \\
\hline 12- AG $1051\left(\mathrm{DH}^{4}\right)$ & $54.1 \mathrm{~d}-\mathrm{f}$ & $68.5 \mathrm{~d}-\mathrm{f}$ & $71.9 \mathrm{~b}-\mathrm{e}$ & $68.5 \mathrm{c}-\mathrm{f}$ & 77.8 a-e & 68.2 \\
\hline 16- BRS $2114(\mathrm{DH})$ & $60.2 b-d$ & $68.8 \mathrm{c}-\mathrm{f}$ & $70.2 \mathrm{c}-\mathrm{f}$ & 72.9 a-e & $67.2 \mathrm{f}-\mathrm{h}$ & 67.9 \\
\hline 15- BRS $2223(\mathrm{DH})$ & $60.4 \mathrm{~b}-\mathrm{d}$ & $74.9 \mathrm{~b}-\mathrm{d}$ & $67.4 \mathrm{~d}-\mathrm{g}$ & $67.6 \mathrm{~d}-\mathrm{f}$ & $67.6 \mathrm{f}-\mathrm{h}$ & 67.6 \\
\hline 14- Traktor (DH) & $59.2 \mathrm{c}-\mathrm{e}$ & $71.2 \mathrm{~b}-\mathrm{e}$ & $64.7 \mathrm{e}-\mathrm{h}$ & $70.2 \mathrm{~b}-\mathrm{e}$ & $72.4 \mathrm{~d}-\mathrm{f}$ & 67.5 \\
\hline 21- BRS 3060 (TH) & $62.5 \mathrm{bc}$ & 65.8 ef & $67.9 \mathrm{~d}-\mathrm{g}$ & $67.3 \mathrm{~d}-\mathrm{f}$ & $70.8 \mathrm{e}-\mathrm{g}$ & 66.9 \\
\hline 19- AGN $3150\left(\mathrm{TH}^{5}\right)$ & $61.2 \mathrm{~b}-\mathrm{d}$ & $63.5 \mathrm{fg}$ & $59.7 \mathrm{~h}$ & $66.2 \mathrm{e}-\mathrm{g}$ & $63.8 \mathrm{gh}$ & 62.9 \\
\hline 22- AG $6690(\mathrm{TH})$ & $61.9 \mathrm{bc}$ & $61.5 \mathrm{fg}$ & $63.0 \mathrm{f}-\mathrm{h}$ & $61.7 \mathrm{fg}$ & $61.8 \mathrm{~h}$ & 62.0 \\
\hline 24- Exceler (TH) & 52.6 ef & $58.4 \mathrm{~g}$ & $66.9 \mathrm{~d}-\mathrm{h}$ & $62.6 \mathrm{fg}$ & $63.0 \mathrm{~h}$ & 60.7 \\
\hline 23- AGN $3180(\mathrm{TH})$ & $51.2 \mathrm{f}$ & $58.6 \mathrm{~g}$ & $60.5 \mathrm{gh}$ & $59.4 \mathrm{~g}$ & $62.3 \mathrm{~h}$ & 58.4 \\
\hline Means & 62.3 & 72.1 & 71.6 & 71.8 & 73.2 & \\
\hline
\end{tabular}

${ }^{1} \mathrm{SH}=$ single cross hybrid; ${ }^{2} \mathrm{MSH}=$ modified $\mathrm{SH} ;{ }^{3}$ Var $=$ variety; ${ }^{4} \mathrm{DH}=$ double cross hybrid; ${ }^{5} \mathrm{TH}=$ three-way cross hybrid. Linear polynomial regression (L) and/or Quadratic (Q) were significant only for cultivars 01, 07, 12, 13, 14, 17 and 20. Test $\mathrm{F}$ (cultivar) = $24.4^{* *} ; \mathrm{F}$ interaction $(\mathrm{Zn}$ vs cult $)=1.68^{*} ; \mathrm{CV} \%(\mathrm{Zn})=1.96 ; \mathrm{CV} \%($ cult $)=5.86$. Means followed by the same letters, in columns, do not differ by Duncan's test $(0.05)$.

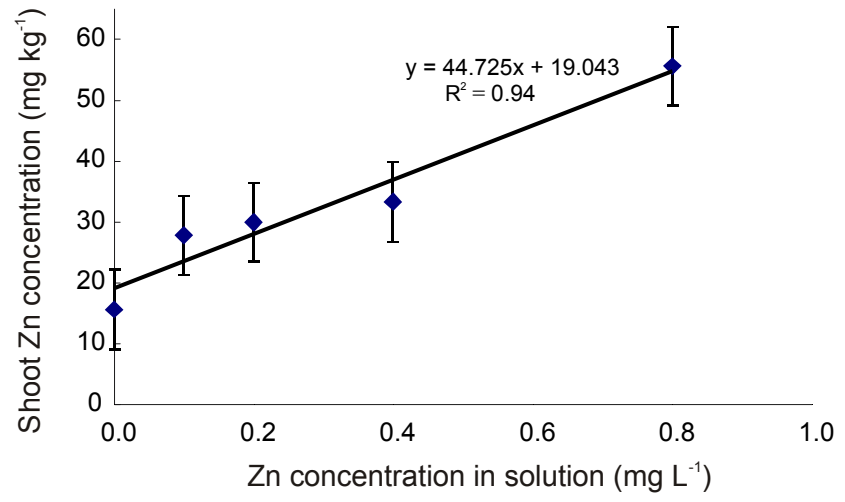

Figure 2 - Shoot $\mathrm{Zn}$ concentrations in 24-day-old maize plants grown under increasing $\mathrm{Zn}$ concentrations (Means of 24 cultivars, 72 observations).

On the other hand, total $\mathrm{Zn}$-shoot content was the parameter that best correlated with plant height $(\mathrm{r}=$ $\left.0.66^{* *}\right)$ and with dry matter yield $\left(\mathrm{r}=0.67^{* *}\right)$, better re- flecting the differences among genotypes (Tables 5 and 7, Figures 3 and 4). The analysis of variance showed highly significant differences for cultivars and their interaction with $\mathrm{Zn}$ concentrations in nutrient solution. The polynomial regression was highly significant for total $\mathrm{Zn}$ shoot content as a function of external $\mathrm{Zn}$ concentration, for all cultivars (Table 5). The 24 cultivar means were significantly adjusted for a quadratic polynome $(\mathrm{R}=$ 0.97).

The nutrient use efficiency index (EI) for the shoot dry matter production according to Siddiqi \& Glass (1981) normally tend to decrease with the nutrient concentration increase in the external media, and this actually occurred to all cultivars in this experiment. This tendency is shown in Figure 5, which refers to the 24 cultivar means. Three-fold differences among cultivars (from 8.59 to 26.42) were obtained for this index in the lowest $\mathrm{Zn}$ treatment, clearly demonstrating the differential plant$\mathrm{Zn}$ demands and efficiencies for $\mathrm{Zn}$ uptake and use. The 
Table 4 - Shoot Zn concentrations of 24-day-old maize plants grown under increasing Zn concentrations.

\begin{tabular}{|c|c|c|c|c|c|c|}
\hline \multirow{2}{*}{$\begin{array}{l}\text { Maize } \\
\text { cultivar }\end{array}$} & \multicolumn{6}{|c|}{$\mathrm{Zn}$ concentrations $\left(\mathrm{mg} \mathrm{L}^{-1}\right)$} \\
\hline & 0.0 & 0.1 & 0.2 & 0.4 & 0.8 & Mean \\
\hline & 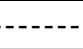 & 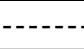 & 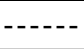 & $-\cdots$ & 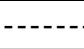 & (n) \\
\hline 19- AGN $3150\left(\mathrm{TH}^{5}\right)$ & 23.2 & 40.1 & 46.2 & 41.4 & 57.0 & $41.6 \mathrm{a}$ \\
\hline 23- AGN $3180(\mathrm{TH})$ & 24.2 & 38.3 & 37.6 & 45.8 & 52.1 & $39.6 \mathrm{ab}$ \\
\hline 22- AG $6690(\mathrm{TH})$ & 24.6 & 36.3 & 37.6 & 42.5 & 55.0 & $39.2 \mathrm{ab}$ \\
\hline 18- XB $8010(\mathrm{DH})$ & 22.1 & 35.0 & 38.9 & 38.0 & 59.7 & $38.7 \mathrm{ab}$ \\
\hline 12- AG $1051\left(\mathrm{DH}^{4}\right)$ & 22.4 & 31.4 & 30.6 & 47.1 & 57.8 & $37.8 \mathrm{bc}$ \\
\hline 24- Exceler $(\mathrm{TH})$ & 22.3 & 33.2 & 28.0 & 38.6 & 52.0 & $34.8 \mathrm{~cd}$ \\
\hline 04- P30F33 (SH) & 18.8 & 29.2 & 28.7 & 37.7 & 57.8 & $34.4 \mathrm{c}-\mathrm{e}$ \\
\hline 14- Traktor $(\mathrm{DH})$ & 20.3 & 30.9 & 31.4 & 36.0 & 53.2 & $34.3 \mathrm{c}-\mathrm{f}$ \\
\hline 16- BRS $2114(\mathrm{DH})$ & 21.8 & 32.9 & 34.3 & 32.7 & 49.3 & $34.2 \mathrm{~d}-\mathrm{f}$ \\
\hline 03- P30K75 (SH) & 19.1 & 28.3 & 28.6 & 37.0 & 56.9 & $34.0 \mathrm{~d}-\mathrm{f}$ \\
\hline 21- BRS $3060(\mathrm{TH})$ & 21.5 & 28.6 & 32.3 & 34.8 & 50.9 & $33.6 \mathrm{~d}-\mathrm{g}$ \\
\hline 11- AL 30 (Var) & 19.2 & 26.0 & 30.6 & 37.8 & 53.3 & $33.4 \mathrm{~d}-\mathrm{g}$ \\
\hline 15- BRS $2223(\mathrm{DH})$ & 20.8 & 27.4 & 31.6 & 34.9 & 52.0 & $33.3 \mathrm{~d}-\mathrm{h}$ \\
\hline 02- P30F80 (SH) & 20.4 & 29.1 & 30.6 & 32.2 & 53.5 & $33.1 \mathrm{~d}-\mathrm{h}$ \\
\hline 08- DOW 8420c (MSH) & 18.3 & 25.8 & 27.8 & 36.3 & 55.9 & $32.8 \mathrm{~d}-\mathrm{h}$ \\
\hline $01-\mathrm{AG} 7575\left(\mathrm{SH}^{1}\right)$ & 15.6 & 27.8 & 30.0 & 33.3 & 55.6 & $32.5 \mathrm{~d}-\mathrm{h}$ \\
\hline 05- AS $1545(\mathrm{SH})$ & 17.3 & 26.6 & 31.2 & 37.2 & 49.0 & $32.3 \mathrm{~d}-\mathrm{h}$ \\
\hline 13- AG $122(\mathrm{DH})$ & 20.7 & 27.4 & 28.8 & 32.9 & 45.5 & $31.0 \mathrm{~d}-\mathrm{i}$ \\
\hline 10- AL 34 (Var) & 22.5 & 20.8 & 26.6 & 32.7 & 50.3 & $30.6 \mathrm{e}-\mathrm{i}$ \\
\hline 17- AGN $2012(\mathrm{DH})$ & 18.0 & 26.0 & 28.4 & 29.9 & 50.0 & $30.5 \mathrm{f}-\mathrm{i}$ \\
\hline 07- AS $1533\left(\mathrm{MSH}^{2}\right)$ & 17.4 & 25.4 & 26.4 & 33.4 & 47.7 & 30.0 ghi \\
\hline 06- Tork (SH) & 16.3 & 26.0 & 26.7 & 34.4 & 45.3 & $29.7 \mathrm{ghi}$ \\
\hline 09- ALBandeirante $\left(\operatorname{Var}^{3}\right)$ & 17.7 & 22.9 & 25.9 & 32.7 & 48.1 & $29.5 \mathrm{hi}$ \\
\hline 20- Master (TH) & 18.6 & 22.9 & 26.8 & 30.5 & 43.2 & $28.4 \mathrm{i}$ \\
\hline Means & 15.6 & 27.8 & 30.0 & 33.3 & 55.6 & \\
\hline
\end{tabular}

${ }^{1} \mathrm{SH}=$ single cross hybrid; ${ }^{2} \mathrm{MSH}=$ modified $\mathrm{SH} ;{ }^{3}$ Var $=$ variety; ${ }^{4} \mathrm{DH}=$ double cross hybrid; ${ }^{5} \mathrm{TH}=$ three-way cross hybrid. Linear Polynomial Regression $(\mathrm{L})(\mathrm{R}=0.97 * *)$ significant for all cultivars. Test $\mathrm{F}$ (cultivar) $=8.74 * * ; \mathrm{F}$ interaction $(\mathrm{Zn}$ vs cult $)=1.12 \mathrm{~ns} ; \mathrm{CV} \%$ $(\mathrm{Zn})=8.22 ; \mathrm{CV} \%$ (cult) $=13.54$. Means followed by the same letters in columns do not differ by Duncan's test $(0.05)$.

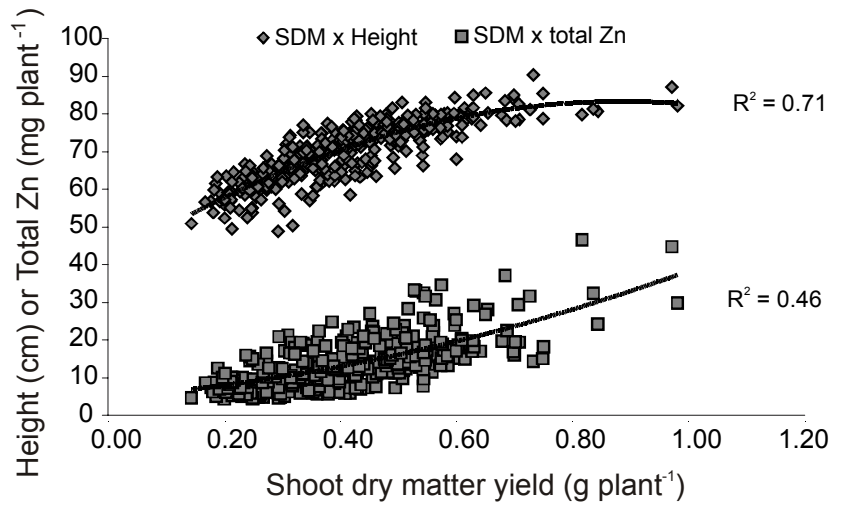

Figure 3 - Relationship between shoot dry matter (SDM) and shoot height $\left(\mathrm{r}=0.81^{* *}\right)$ (a) and between dry matter and total $\mathrm{Zn}$ shoot content $\left(\mathrm{r}=0.67^{* *}\right)(\mathrm{b})$, for 24-day-old maize plants grown in nutrient solution with five $\mathrm{Zn}$ concentrations (total of 360 observations).

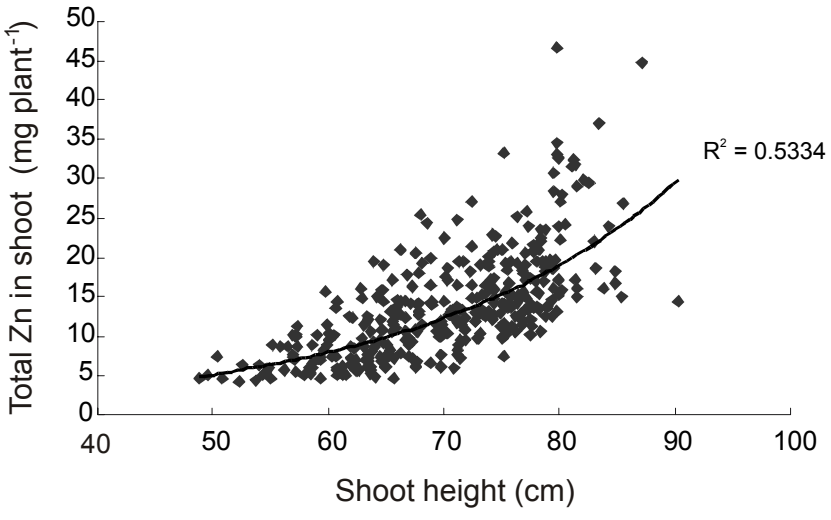

Figure 4 - Relationship between shoot height and total $\mathrm{Zn}$ shoot content (simple correlation coefficient, $r=$ $\left.0.66^{* *}\right)$, for 24-day-old maize plants grown in nutrient solution with five $\mathrm{Zn}$ concentrations (total of 360 observations).

Sci. Agric. (Piracicaba, Braz.), v.62, n.3, p.264-273, May/June 2005 
Table 5 - Total $\mathrm{Zn}$ shoot content in 24-day-old maize plants grown under increasing $\mathrm{Zn}$ concentrations.

\begin{tabular}{|c|c|c|c|c|c|c|}
\hline \multirow{2}{*}{$\begin{array}{l}\text { Maize } \\
\text { cultivar }\end{array}$} & \multicolumn{6}{|c|}{ Zn concentrations $\left(\mathrm{mg} \mathrm{L}^{-1}\right)$} \\
\hline & 0.0 & 0.1 & 0.2 & 0.4 & 0.8 & Mean \\
\hline & 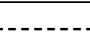 & & $-\ldots-m$ & $t^{-1}-\ldots-$ & 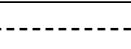 & $-1-$ \\
\hline 19- AGN $3150\left(\mathrm{TH}^{5}\right)$ & $5.6 \mathrm{a}$ & 9.8 a-d & $9.6 \mathrm{bc}$ & $11.3 \mathrm{e}$ & $13.2 \mathrm{i}$ & 9.9 \\
\hline 23- AGN $3180(\mathrm{TH})$ & $4.5 \mathrm{a}$ & $7.6 \mathrm{~cd}$ & $9.4 \mathrm{c}$ & $11.3 \mathrm{e}$ & $14.7 \mathrm{hi}$ & 9.5 \\
\hline 12- AG $1051\left(\mathrm{DH}^{4}\right)$ & $6.8 \mathrm{a}$ & $12.5 \mathrm{a}-\mathrm{d}$ & $16.6 \mathrm{a}$ & $18.8 \mathrm{a}-\mathrm{c}$ & $34.3 \mathrm{a}$ & 17.8 \\
\hline 10- AL 34 (Var) & $9.5 \mathrm{a}$ & $15.1 \mathrm{a}$ & $16.2 \mathrm{a}$ & $17.7 \mathrm{a}-\mathrm{e}$ & $29.0 \mathrm{a}-\mathrm{c}$ & 17.5 \\
\hline 17- AGN 2012 (DH) & $6.9 \mathrm{a}$ & $12.5 \mathrm{a}-\mathrm{d}$ & $14.7 \mathrm{a}-\mathrm{c}$ & $17.6 \mathrm{a}-\mathrm{e}$ & $31.7 \mathrm{ab}$ & 16.7 \\
\hline 06- Tork (SH) & $6.2 \mathrm{a}$ & $14.3 \mathrm{ab}$ & $15.3 \mathrm{a}-\mathrm{c}$ & $17.9 \mathrm{a}-\mathrm{d}$ & $28.0 \mathrm{~b}-\mathrm{d}$ & 16.4 \\
\hline 03- P30K75 (SH) & $7.8 \mathrm{a}$ & $12.2 \mathrm{a}-\mathrm{d}$ & $16.0 \mathrm{ab}$ & $18.7 \mathrm{a}-\mathrm{c}$ & $26.4 \mathrm{~b}-\mathrm{d}$ & 16.2 \\
\hline 04- P30F33 (SH) & $8.5 \mathrm{a}$ & $14.6 \mathrm{ab}$ & $16.2 \mathrm{a}$ & $16.8 \mathrm{a}-\mathrm{e}$ & $25.0 \mathrm{c}-\mathrm{e}$ & 16.2 \\
\hline 07- AS $1533\left(\mathrm{MSH}^{2}\right)$ & $7.9 \mathrm{a}$ & $13.8 \mathrm{a}-\mathrm{c}$ & $16.7 \mathrm{a}$ & $20.2 \mathrm{ab}$ & $22.3 \mathrm{~d}-\mathrm{g}$ & 16.2 \\
\hline 13- AG $122(\mathrm{DH})$ & $5.6 \mathrm{a}$ & $11.9 \mathrm{a}-\mathrm{d}$ & $12.8 \mathrm{a}-\mathrm{c}$ & $22.8 \mathrm{a}$ & $22.3 \mathrm{~d}-\mathrm{g}$ & 15.1 \\
\hline $01-\mathrm{AG} 7575\left(\mathrm{SH}^{1}\right)$ & $6.0 \mathrm{a}$ & $13.2 \mathrm{a}-\mathrm{d}$ & $13.5 \mathrm{a}-\mathrm{c}$ & 18.9 a-c & $23.1 \mathrm{c}-\mathrm{f}$ & 15.0 \\
\hline 20- Master (TH) & $6.8 \mathrm{a}$ & $12.2 \mathrm{a}-\mathrm{d}$ & $12.7 \mathrm{a}-\mathrm{c}$ & $16.3 \mathrm{~b}-\mathrm{e}$ & $26.1 \mathrm{~b}-\mathrm{d}$ & 14.8 \\
\hline 11- AL 30 (Var) & $7.3 \mathrm{a}$ & $11.7 \mathrm{a}-\mathrm{d}$ & $15.8 \mathrm{a}-\mathrm{c}$ & $13.2 \mathrm{c}-\mathrm{e}$ & $23.6 \mathrm{c}-\mathrm{f}$ & 14.3 \\
\hline 14- Traktor (DH) & $5.4 \mathrm{a}$ & $11.4 \mathrm{a}-\mathrm{d}$ & $11.1 \mathrm{a}-\mathrm{c}$ & $15.6 \mathrm{~b}-\mathrm{e}$ & $27.7 b-d$ & 14.2 \\
\hline 09- ALBandeirante $\left(\operatorname{Var}^{3}\right)$ & $6.4 \mathrm{a}$ & 10.8 a-d & $14.5 \mathrm{a}-\mathrm{c}$ & $16.1 \mathrm{~b}-\mathrm{e}$ & $22.7 \mathrm{~d}-\mathrm{g}$ & 14.1 \\
\hline 02- P30F80 (SH) & $8.4 \mathrm{a}$ & $12.0 \mathrm{a}-\mathrm{d}$ & $13.1 \mathrm{a}-\mathrm{c}$ & $15.5 \mathrm{~b}-\mathrm{e}$ & $19.8 \mathrm{e}-\mathrm{h}$ & 13.8 \\
\hline 08- DOW 8420c (MSH) & $6.6 \mathrm{a}$ & $10.6 \mathrm{a}-\mathrm{d}$ & $13.3 \mathrm{a}-\mathrm{c}$ & $15.8 \mathrm{~b}-\mathrm{e}$ & $22.4 \mathrm{~d}-\mathrm{g}$ & 13.8 \\
\hline 18- XB $8010(\mathrm{DH})$ & $5.3 \mathrm{a}$ & 10.9 a-d & $10.8 \mathrm{a}-\mathrm{c}$ & $15.3 \mathrm{~b}-\mathrm{e}$ & $19.7 \mathrm{e}-\mathrm{h}$ & 12.4 \\
\hline 05- AS 1545 (SH) & $5.2 \mathrm{a}$ & 9.9 a-d & $12.3 \mathrm{a}-\mathrm{c}$ & $13.9 \mathrm{~b}-\mathrm{e}$ & $19.0 \mathrm{e}-\mathrm{i}$ & 12.0 \\
\hline 15- BRS $2223(\mathrm{DH})$ & $6.3 \mathrm{a}$ & $11.0 \mathrm{a}-\mathrm{d}$ & $11.1 \mathrm{a}-\mathrm{c}$ & $12.8 \mathrm{c}-\mathrm{e}$ & $18.8 \mathrm{e}-\mathrm{i}$ & 12.0 \\
\hline 22- AG $6690(\mathrm{TH})$ & $7.6 \mathrm{a}$ & 8.8 a-d & $12.2 \mathrm{a}-\mathrm{c}$ & $13.3 \mathrm{c}-\mathrm{e}$ & $16.7 \mathrm{~g}-\mathrm{i}$ & 11.7 \\
\hline 21- BRS $3060(\mathrm{TH})$ & $6.7 \mathrm{a}$ & $8.4 \mathrm{~b}-\mathrm{d}$ & $10.2 \mathrm{a}-\mathrm{c}$ & $11.7 \mathrm{de}$ & $18.6 \mathrm{f}-\mathrm{i}$ & 11.1 \\
\hline 16- BRS $2114(\mathrm{DH})$ & $5.3 \mathrm{a}$ & 9.9 a-d & $11.2 \mathrm{a}-\mathrm{c}$ & $13.5 \mathrm{c}-\mathrm{e}$ & $15.3 \mathrm{hi}$ & 11.0 \\
\hline 24- Exceler (TH) & $5.0 \mathrm{a}$ & $7.0 \mathrm{~d}$ & $11.1 \mathrm{a}-\mathrm{c}$ & $12.0 \mathrm{de}$ & $18.6 \mathrm{f}-\mathrm{i}$ & 10.8 \\
\hline Means & 6.6 & 11.3 & 13.2 & 15.7 & 22.5 & \\
\hline
\end{tabular}

${ }^{1} \mathrm{SH}=$ single cross hybrid; ${ }^{2} \mathrm{MSH}=$ modified SH; ${ }^{3} \mathrm{Var}=$ variety; ${ }^{4} \mathrm{DH}=$ double cross hybrid; ${ }^{5} \mathrm{TH}=$ three-way cross hybrid. Linear Polynomial Regression $(\mathrm{L})$ presented significant $\left(\mathrm{R}^{* *}\right)$ for all cultivars. Test $\mathrm{F}$ (cultivar) $=8.42 * *$; $\mathrm{F}$ interaction $(\mathrm{Zn}$ vs cult) $=1.42 *$; $\mathrm{CV} \%(\mathrm{Zn})=5.69 ; \mathrm{CV} \%$ (cult) $=23.46$. Means followed by the same letters, in columns, do not differ by Duncan's test $(0.05)$.

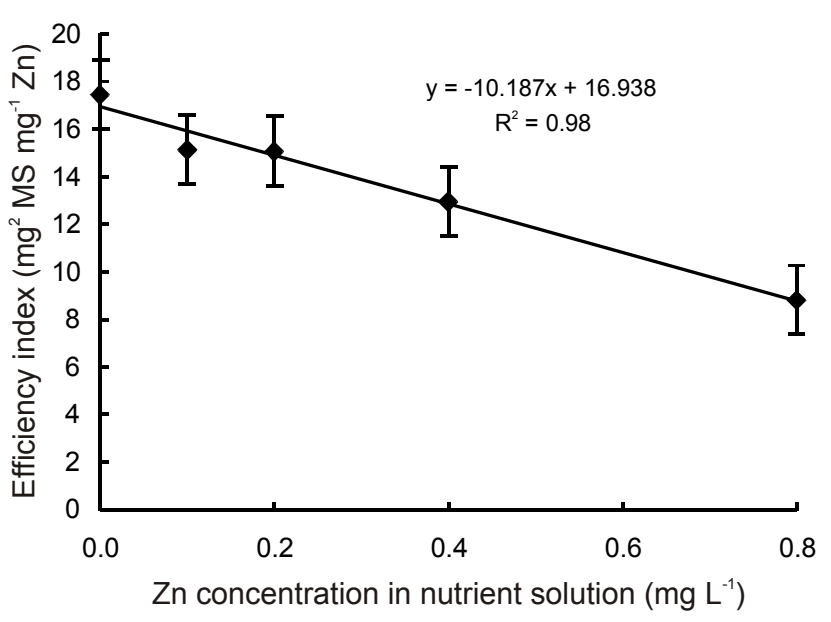

Figure 5 - Efficiency index in maize plants (dry matter yield per unity of $\mathrm{Zn}$ shoot concentration) as a function of $\mathrm{Zn}$ concentrations in solution. Means over 24 cultivars $(72$ observations). efficiency index variation was probably influenced by differential maize cultivar abilities in using $\mathrm{Zn}$ for germination and growth, once no correlation was found between seed $\mathrm{Zn}$ concentration and EI in the free-Zn treatment. Additionally, they were not apparently related to the crossing-type material (simple hybrid, double or threeway cross, or variety).

Genotypes may present different mechanisms for Zn efficiency. Welch (1999) emphasized the importance of $\mathrm{Zn}$ grain reserves on early wheat seedling performance, mainly under low external $\mathrm{Zn}$ conditions and that the benefits of $\mathrm{Zn}$ grain reserves conferring seedling vigor during germination cannot be substituted by $\mathrm{Zn}$ supply after germination. Greater $\mathrm{Zn}$ grain reserves resulted in greater seedling root and shoot growth in a $\mathrm{Zn}$ deficient soil and the differences between plants from high-Zn and low-Zngrains were evident even after six weeks of growth (Welch, 1999). Nevertheless, despite showing the same tissue or grain $\mathrm{Zn}$ concentrations, efficient cultivars may 
differ from inefficient ones by their ability of expressing Zn-efficiency, only at low Zn levels, by maintaining physiological processes and higher enzyme activities (carbonic anhydrase and others). At sufficient $\mathrm{Zn}$ levels, differences in enzyme activities between Zn-efficient and Zn-inefficient cultivars may not appear (Rengel, 1999).

In order to evaluate and classify the cultivars as to their efficiency in $\mathrm{Zn}$ uptake and use under low $\mathrm{Zn}$ and to their responsiveness to $\mathrm{Zn}$ supply, values of efficiency index (EI) at low $\mathrm{Zn}$ were plotted against the relative increases in dry matter production, that is, the ratio of maximum/minimum dry matter yield (DMmax/DMmin) (Figure 6). The overall mean for the 24 cultivars, in each axis, allowed to classify them in: efficient-responsive (ER) = 01 (AG 7575); 06 (Tork); 09 (AL-Bandeirante); 10 (AL 34); 17 (AGN 2012); 20 (Master); efficient-non-responsive $(\mathrm{ENR})=02(\mathrm{P} 30 \mathrm{~F} 80) ; 03(\mathrm{~F} 30 \mathrm{~K} 75) ; 04(\mathrm{P} 30 \mathrm{~F} 33)$;
07 (AS 1533); 08 (DOW 8420); 11 (AL 30); inefficientresponsive $(\mathrm{IR})=12(\mathrm{AG} 1051) ; 13(\mathrm{AG} \mathrm{122}) ; 14$ (Traktor); 16 (BRS 2114); 18 (XB 8010); 23 (AGN 3180); 24 (Exceler); and inefficient-non-responsive (INR) $=05$ (AS 1545); 15 (BRS 2223); 19 (AGN 3150); 21 (BRS 3060); 22 (AG 6690). Therefore, twelve cultivars were considered efficient, with EI values above the average $\left(17.5 \mathrm{mg}^{2} \mathrm{DM} \mathrm{mg}^{-1} \mathrm{Zn}\right)$ and twelve were inefficient. Responsive cultivars presented relative increases in dry matter production above de average (DMmax/DMmin ratio $>1.53$ ) (Figure 6).

Nutrient concentrations $(\mathrm{K}, \mathrm{P}, \mathrm{Ca}, \mathrm{Mg}, \mathrm{Cu}, \mathrm{Fe}$ and $\mathrm{Mn}$ ) in plant shoot were within normal limits (Table 6) for young maize plants. $\mathrm{Zn}$ concentrations in plants were positively correlated with $\mathrm{K}\left(\mathrm{r}=0.27^{* *}\right)$ and $\mathrm{Ca}(\mathrm{r}=$ $\left.0.25^{* *}\right)$, that is, there was a tendency to increase the $\mathrm{K}$ and $\mathrm{Ca}$ concentrations in plants in function of $\mathrm{Zn}$ con-

Table 6 - Shoot K, P, Ca, Mg, Cu, Fe and Mn concentrations of 24-day-old maize plants grown under increasing $\mathrm{Zn}$ concentrations (means over 72 observations and 24 cultivars).

\begin{tabular}{lrrrrrr}
\hline \multirow{2}{*}{ Nutrient } & \multicolumn{5}{c}{ Zn concentrations $\left(\mathrm{mg} \mathrm{L}^{-1}\right)$} \\
\cline { 2 - 7 } & 0.0 & 0.1 & 0.2 & 0.4 & 0.8 & Mean \\
\hline $\mathrm{K}\left(\mathrm{g} \mathrm{kg}^{-1}\right)\left(^{(}\right)$ & 50.6 & 51.2 & 51.7 & 52.3 & 52.0 & 51.5 \\
$\mathrm{P}\left(\mathrm{g} \mathrm{kg}^{-1}\right)\left(^{2}\right)$ & 6.4 & 5.4 & 4.9 & 4.7 & 4.8 & 5.2 \\
$\mathrm{Ca}\left(\mathrm{g} \mathrm{kg}^{-1}\right)\left(^{3}\right)$ & 16.0 & 17.0 & 18.7 & 17.8 & 18.2 & 15.3 \\
$\mathrm{Mg}\left(\mathrm{g} \mathrm{kg}^{-1}\right)\left(^{4}\right)$ & 5.2 & 5.6 & 5.2 & 5.3 & 4.3 & 4.8 \\
$\mathrm{Cu}\left(\mathrm{mg} \mathrm{kg}^{-1}\right)\left(^{5}\right)$ & 12.8 & 11.6 & 10.6 & 9.4 & 9.9 & 10.9 \\
$\mathrm{Fe}\left(\mathrm{mg} \mathrm{kg}^{-1}\right)\left(^{6}\right)$ & 235.1 & 171.7 & 159.0 & 152.0 & 140.5 & 171.6 \\
$\mathrm{Mn}\left(\mathrm{mg} \mathrm{kg}^{-1}\right)\left(^{7}\right)$ & 143.5 & 127.5 & 117.4 & 111.6 & 124.9 & 125.0 \\
\hline
\end{tabular}

${ }^{1}$ Polynomial Regression not significant for all cultivars. Test $\mathrm{F}$ (cultivar) $=8.69$ **; F interaction $(\mathrm{Zn}$ vs cult $)=1.02 \mathrm{~ns} ; \mathrm{CV} \%(\mathrm{Zn})=7.12$; $\mathrm{CV} \%$ (cult) $=6.93$. ${ }^{2}$ Polynomial Regression not significant for all cultivars. Test $\mathrm{F}(\mathrm{Zn})=6.03^{*}$. Test $\mathrm{F}$ (cultivar) $=11.45^{* *} ; \mathrm{F}$ interaction $(\mathrm{Zn}$ vs cult $)=1.59^{* *} ; \mathrm{CV} \%(\mathrm{Zn})=9.70 ; \mathrm{CV} \%$ (cult) $=12.35 .{ }^{3}$ Polynomial Regression for Zn levels not significant for all cultivars. Test $\mathrm{F}(\mathrm{Zn})=4.88^{*}$. Test $\mathrm{F}$ (cultivar) $=20.49^{* *} ; \mathrm{F}$ interaction $(\mathrm{Zn}$ vs cult $)=1.59^{* *}$; CV\% $(\mathrm{Zn})=4.09 ; \mathrm{CV} \%$ (cult) $=8.96 .{ }^{4} \mathrm{Polynomial}$ Regression for $\mathrm{Zn}$ levels not significant for all cultivars. Test $\mathrm{F}(\mathrm{Zn})=18.59^{* *}$. Test $\mathrm{F}$ (cultivar) $=10.21^{* *}$; $\mathrm{F}$ interaction $(\mathrm{Zn}$ vs cult) $=$ $0.92 \mathrm{~ns} ; \mathrm{CV} \%(\mathrm{Zn})=2.13$; CV\% (cult) $=9.84$. ${ }^{5}$ Polynomial Regression not significant for all cultivars. Test $\mathrm{F}(\mathrm{Zn})=10.53^{* *}$. Test $\mathrm{F}$ $($ cultivar $)=8.69 * ;$ F interaction $(\mathrm{Zn}$ vs cult $)=1.51 * * ; \mathrm{CV} \%(\mathrm{Zn})=7.26 ; \mathrm{CV} \%($ cult $)=12.94 .{ }^{6} \mathrm{Quadratic}$ Polynomial Regression $\left(\mathrm{R}^{*}\right)$ for cultivars $1,10,13,14,16,17,20,21$ and 22 . Test $\mathrm{F}$ (cultivar) $=3.65^{* *} ; \mathrm{F}$ interaction $(\mathrm{Zn}$ vs cult $)=1.88^{* *} ; \mathrm{CV} \%(\mathrm{Zn})=5.78 ; \mathrm{CV} \%$ $($ cult $)=13.77 .{ }^{7}$ Quadratic Polynomial Regression $\left(\mathrm{R}^{*}\right)$ for cultivar means. Test $\mathrm{F}(\mathrm{Zn})=16.67^{* *}$. Test $\mathrm{F}($ cultivar $)=14.42^{* *} ; \mathrm{F}$ interaction $(\mathrm{Zn}$ vs cult $)=1.14 \mathrm{~ns} ; \mathrm{CV} \%(\mathrm{Zn})=7.25 ; \mathrm{CV} \%$ (cult) $=11.25$.

Table 7 - Simple correlations (r) between the variables evaluated in 24-day-old maize plants grown under increasing $\mathrm{Zn}$ concentrations (pairs of 360 observations).

\begin{tabular}{|c|c|c|c|c|c|c|c|c|c|c|}
\hline & Height & TotZn & $\mathrm{Zn}$ & $\mathrm{K}$ & $\mathrm{P}$ & $\mathrm{Ca}$ & $\mathrm{Mg}$ & $\mathrm{Cu}$ & $\mathrm{Fe}$ & $\mathrm{Mn}$ \\
\hline SDM & $0.81 * *$ & $0.67 * *$ & $-0.10 *$ & $-0.43 * *$ & $-0.70 * *$ & ns & $0.41 * *$ & $-0.28 * *$ & $-0.45 * *$ & $-0.34 * *$ \\
\hline Height & --- & $0.66 * *$ & $0.10 *$ & $-0.29 * *$ & $-0.69 * *$ & ns & $0.46^{* *}$ & $-0.35 * *$ & $-0.58 * *$ & $-0.27 * *$ \\
\hline $\mathrm{Zn}$ & --- & --- & --- & $0.27 * *$ & $-0.11 *$ & $0.25 * *$ & $-0.37 * *$ & $-0.24 * *$ & $-0.40 * *$ & ns \\
\hline K & --- & --- & --- & --- & $0.38 * *$ & ns & $-0.29 * *$ & ns & ns & ns \\
\hline $\mathrm{P}$ & --- & --- & --- &.-- & --- & ns & $-0.30 * *$ & $0.53 * *$ & $0.65 * *$ & $0.58 * *$ \\
\hline $\mathrm{Ca}$ & --- & --- & --- & --- & --- & --- & $0.18 * *$ & ns & ns & $0.40 * *$ \\
\hline $\mathrm{Mg}$ & --- & --- & --- & --- & --- & --- & --- & $0.12 *$ & ns & $0.14 * *$ \\
\hline $\mathrm{Cu}$ & --- & --- & --- & -- & --- & --- & --- & --- & $0.67 * *$ & $0.61 * *$ \\
\hline $\mathrm{Fe}$ & --- & --- & --- & --- & --- & --- & --- & --- & --- & $0.55 * *$ \\
\hline
\end{tabular}

Sci. Agric. (Piracicaba, Braz.), v.62, n.3, p.264-273, May/June 2005 


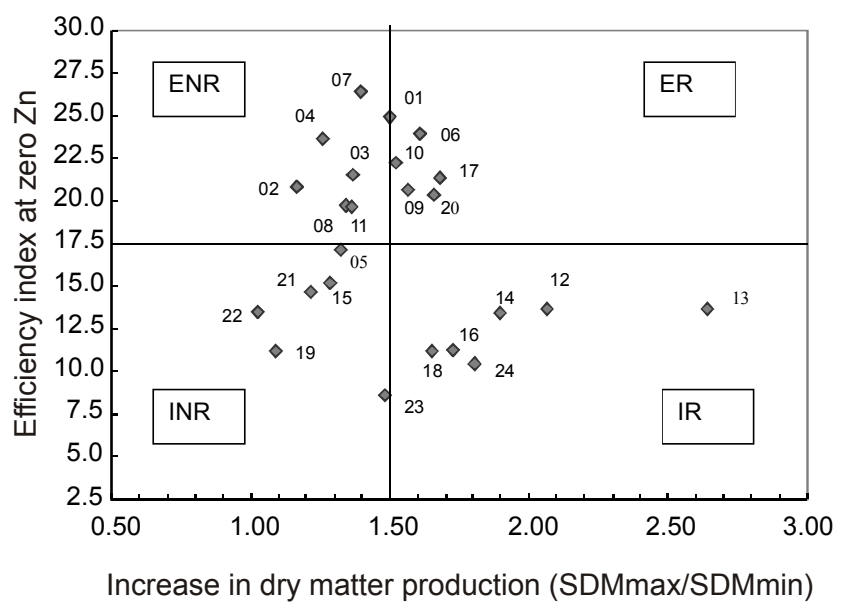

Figure 6 - Classification of maize cultivars: $y$-axis = efficiency index $\left(\mathrm{mg}^{2} \mathrm{DM} \mathrm{mg}^{-1} \mathrm{Zn}\right)$ for $\mathrm{Zn}$ utilization at the lowest $\mathrm{Zn}$ rate in solution $\left(0.0 \mathrm{mg} \mathrm{L}^{-1}\right)$; $\mathrm{x}$-axis = relative increase in shoot dry matter $(\mathrm{SDM})$ in response to $\mathrm{Zn}$ rates $(\mathrm{SDM}$ maximum/minimum): Efficient -Responsive $(\mathrm{ER})=01$ (AG 7575), 06 (Tork), 09 (AL Bandeirante), 10 (AL 34), 17 (AGN 2012) and 20 (Master); Efficient-nonResponsive $(\mathrm{ENR})=02(\mathrm{P} 30 \mathrm{~F} 80), 03$ (P30K75), 04 (P30F33), 07 (AS 1533), 08 (DOW 8420) and 11 (AL 30); Inefficient-Responsive (IR) $=12$ (AG 1051), 13 (AG 122), 14 (Traktor), 16 (BRS 2114), 18 (XB 8010), 23 (AGN 3180) and 24 (Exceler); and Inefficient-nonresponsive $($ INR) $=05$ (AS 1545), 15 (BRS 2223), 19 (AGN 3150), 21 (BRS 3060) and 22 (AG 6690).

centration in the external media and $\mathrm{Zn}$ concentration in plants. These positive correlations might be due to beneficial effect of $\mathrm{Zn}$ to roots increasing the uptake of those nutrients. In contrast, $\mathrm{Zn}$ concentration in plants demonstrated a negative correlation with $\mathrm{P}\left(\mathrm{r}=-0.11^{*}\right), \mathrm{Mg}(\mathrm{r}$ $\left.=-0.37^{* *}\right), \mathrm{Cu}\left(\mathrm{r}=-0.24^{* *}\right)$ and $\mathrm{Fe}\left(\mathrm{r}=-0.40^{* *}\right)$ contents in plants. There was a tendency to decrease shoot $\mathrm{P}, \mathrm{Mg}, \mathrm{Cu}$ and $\mathrm{Fe}$ concentrations with increasing external $\mathrm{Zn}$ and $\mathrm{Zn}$ plant contents. In the case of $\mathrm{P}$, this negative correlation might be due to the formation of insoluble zinc phosphate in the rhizosphere. Nevertheless, for $\mathrm{Mg}$, $\mathrm{Cu}$ and $\mathrm{Fe}$ there was a higher competition with $\mathrm{Zn}$ for sites of uptake by roots, what became more evident with the $\mathrm{Zn}$ increase in the nutrient solution (Tables 6 and 7). The competition among bivalent cations for the carrier binding sites in the plasma membrane is well documented (Marschner, 1995) and usually when the external concentration of one of them increases, a significant reduction in the concentration of other bivalent cations in plant tissue is observed.

Differences among cultivars were small, although significant, for $\mathrm{K}$ (46.4 - $\left.57.8 \mathrm{~g} \mathrm{~kg}^{-1}\right)$; $\mathrm{P}$ (4.0 $\left.6.2 \mathrm{~g} \mathrm{~kg}^{-1}\right)$; $\mathrm{Ca}\left(11.7\right.$ - $\left.17.7 \mathrm{~g} \mathrm{~kg}^{-1}\right) ; \mathrm{Mg}\left(3.9\right.$ - $\left.5.5 \mathrm{~g} \mathrm{~kg}^{-1}\right)$; $\mathrm{Cu}\left(8.3\right.$ - $\left.12.6 \mathrm{mg} \mathrm{kg}^{-1}\right)$; and Fe contents (152 - $191 \mathrm{mg}$ $\left.\mathrm{kg}^{-1}\right)$, considering that all materials were submitted to the same concentrations of these nutrients ( $F$ test significant for cultivars, Table 7). The variation in nutrient concentrations among the cultivars could be due to a dilution effect related to higher or lower dry matter yield. This is evidenced by the significant negative correlations between shoot dry matter yield and contents of $\mathrm{K}, \mathrm{P}, \mathrm{Cu}, \mathrm{Fe}$ and $\mathrm{Mn}$; and also betwe-en plant height and these nutrient concentrations (Table 6).

The technique used for the evaluation of young plants in nutrient solution allowed differentiating maize cultivars as to their efficiency in $\mathrm{Zn}$ uptake and use. The genotype responses were evaluated in 24-day-old plants for their dry matter yields, plant heights and total $\mathrm{Zn}$ contents in shoot parts. Twelve out of 24 cultivars were efficient at low $\mathrm{Zn}$ rates and six of them were responsive to increasing $\mathrm{Zn}$ supply in nutrient solution. Grain reserves were apparently enough to provide $\mathrm{Zn}$ for good germination and initial seedling growth. However, the variation in $\mathrm{Zn}$ efficiencies observed among cultivars might be attributed to differential physiological mechanisms, conferring abilities in utilizing $\mathrm{Zn}$ for germination and initial seedling growth that show up especially under Zndeficiency, characterizing plant adaptation to low $\mathrm{Zn} \mathrm{lev-}$ els.

Cultivars presenting higher relative dry matter increases (DMmax/DMmin) in response to $\mathrm{Zn}$ supply presented also low Zn-efficiency indexes (Zn-inefficient-responsive cultivars: $12=\mathrm{AG} 1051,13=\mathrm{AG} \mathrm{122;14}=$ Traktor, 24= Exceler), evidencing to be high $\mathrm{Zn}$ demander plants.

Corn cultivars have been extensively tested in the State of São Paulo during several years (Duarte \& Paterniani, 2000a). Cultivars AG 1051, AG 122; Traktor and Exceler are well-adapted and high yielding cultivars. Exceler, a highly Al-susceptible cultivar, has shown good field performance in acid soils, what probably indicated P-efficiency characters. The Zn-inefficient-non-responsive cultivars 19 (AGN 3150) and 23 (AGN 3180) have presented good potential yielding only in restricted areas. Among the Zn-efficient cultivars AGN 2012 has shown an expressive behavior and scored in $8^{\text {th }}$ out of 24 top materials. Tork and Master (Zn-efficient-responsive cultivars) are high yielding genotypes in acid soils (around 8,000 and $8,500 \mathrm{~kg} \mathrm{ha}^{-1}$, respectively), but Al-susceptible genotypes, and the latter also show high yield stability in the whole State. P30K75 and P30F33 (Zn-efficientnon-responsive cultivars) performed better in acid soils than in non-acid ones (P30F33 had 20\% higher yield average of $8,596 \mathrm{~kg} \mathrm{ha}^{-1}$ - in acid as compared to nonacid soils), evidencing highly adapted genotypes (Duarte \& Paterniani, 2000b).

\section{ACKNOWLEDGEMENTS}

To FAPESP and CNPq for financial support and fellowships, and to Patrícia Heloá dos Santos for technical support. 


\section{REFERENCES}

BITYUTSKII, N.P.; MAGNITKSKIY, S.V.; KOROBEYNIKOVA, L.P.; SHCHIPAREV, S.M.; TERLEEV, V.V.; MATVEEVA, G.V. Metal content in the various parts of maize kernels as related to maize root growth during germination. Russian Journal of Plant Physiology, v.46, p.426$430,1999$.

BITYUTSKII, N.P.; MAGNITKSKIY, S.V.; KOROBEYNIKOVA, L.P.; LUKINA, E.I.; SOLOVIOVA, A.N.; PATSEVITCH, V.G.; LAPSHINA,

I.N. Micronutrient and calcium distribution in germinating maize kernels. Russian Journal of Plant Physiology, v.47, p.242-248, 2000.

BITYUTSKII, N.P.; MAGNITKSKIY, S.V.; KOROBEYNIKOVA, L.P.; LUKINA, E.I.; SOLOVIOVA, A.N.; PATSEVITCH, V.G.; LAPSHINA, I.N.; MATVEEVA, G.V. Distribution of iron, manganese, and zinc in mature grain and their mobilization during germination and early seedling development in maize. Journal of Plant Nutrition, v.25, p.635-653, 2002.

BROWN, J.C.; AMBLER, J.E.; CHANEY, R.L.; FOY, C.D. Differential responses of plant genotypes to micronutrients. In: MORTVEDT, J.J.; GIORDANO, P.M.; LINDSAY, W.L. (Ed.) Micronutrients in agriculture. Madison: SSSA, 1972. p.389-418.

CAKMAK, I.; TORUN, B.; ERENOGLU, B.; OZTURK, L.; MARSCHNER, H. KALAYCI, M.; EKIZ, H.; YILMAZ, A. Morphological and physiological differences in the response of cereals to zinc deficiency. Euphytica, v.100, p.349-357, 1998.

CAKMAK, I.; KALAYCI, C.; EKIZ, H.; BRAUN, H.J.; KILINE, Y.; YILMAZ, A. Zinc deficiency as a practical problem in plant and human nutrition in Turkey: A Nato-science for stability project. Field Crops Research, v.60, p.175-188, 1999.

CLARK, R.B. Differential response of maize inbreds to Zn. Agronomy Journal, v.70, p.1057-1060, 1978.

DUARTE, A.P.; PATERNIANI, M.E.A.G.Z. Reflexos da estratificação de ambientes na adaptação e estabilidade da produção de grãos em cultivares de milho. In: DUARTE, A.P.; PATERNIANI, M.E.A.G.Z. (Ed.) Fatores bióticos e abióticos em cultivares de milho e estratificação ambiental: avaliação IAC/CATI/Empresas - 1999/2000. Campinas: Instituto Agronômico, 2000a. p.139-150. (Série Pesquisa APTA, Boletim Científico, 5).

DUARTE, A.P.; PATERniAnI, M.E.A.G.Z. (Ed.) Fatores bióticos e abióticos em cultivares de milho e estratificação ambiental: avaliação IAC/CATI/Empresas - 1999/2000. Campinas: Instituto Agronômico, 2000b. 150p. (Série Pesquisa APTA, Boletim Científico 05).

ERENOGLU, B.; CAKMAK, I.; ROMHELD, V.; DERICI, R.; RENGEL, Z. Uptake of zinc by rye, bread wheat and durum wheat cultivars differing in zinc efficiency. Plant and Soil, v.209, p.245-252, 1999.

FAGERIA, N.K. Screening method of lowland rice genotypes for zinc uptake efficiency. Scientia Agricola, v.58, p.623-626, 2001.

FURLANI, A.M.C.; FURLANI, P.R. Composição e pH de soluções nutritivas para estudos fisiológicos e seleção de plantas em condições nutricionais adversas. Campinas: Instituto Agronômico, 1988. 34p. (Boletim Técnico, 121).

HOPKINS, B.G.; WHITNEY, D.A.; LAMOND, R.E.; JOLLEY, V.D. Phytosiderophore release by sorghum, wheat, and corn under zinc deficiency, Journal of Plant Nutrition, v.21, p.2623-2637, 1998,
KUZ'-MENKO, L.M.; ZHMURKO, M.G.; SIVAK, L.A.; YERMAK, M.M.; DEMCHENKO, T.I.; ERMAK, M.M. Reaction of maize genotypes to zinc application. Fiziologiya i Biokhimiya Kul'turnykh Rastenii, v.26, p.151-155, 1994.

MARSCHNER, H. Mineral nutrition of higher plants. 2.ed. London: Academic Press, 1995. 889p.

PEARSON, J.N.; RENGEL, Z. Uptake and distribution of Zn-65 and Mn54 in wheat grown at sufficient and deficient levels of $\mathrm{Zn}$ and $\mathrm{Mn} .1$. During vegetative growth. Journal of Experimental Botany, v.46, p.833-839, 1995a.

PEARSON, J.N.; RENGEL, Z. Uptake and distribution of Zn-65 and Mn54 in wheat grown at sufficient and deficient levels of $\mathrm{Zn}$ and Mn. 2. During grain development. Journal of Experimental Botany, v.46, p.841-845, 1995b.

PEARSON, J.N.; RENGEL, Z.; GRAHAM, R.D. Regulation of zinc and manganese transport into developing wheat grains having different zinc and manganese concentrations. Journal of Plant Nutrition, v.22, p.1141-1152, 1999.

PEARSON, J.N.; JENNER, C.F.; RENGEL, Z.; GRAHAM, R.D. Differential transport of $\mathrm{Zn}, \mathrm{Mn}$ and sucrose along the longitudinal axis of developing wheat grains. Physiologia Plantarum, v.97, p.332-338, 1996 .

PEARSON, J.N.; RENGEL, Z.; JENNER, C.F.; GRAHAM, R.D. Manipulation of xylem transport affects $\mathrm{Zn}$ and $\mathrm{Mn}$ transport into developing wheat grains of cultured ears. Physiologia Plantarum, v.98, p.229-234, 1996b.

PEARSON, J.N.; RENGEL, Z.; JENNER, C.F.; GRAHAM, R.D. Dynamics of zinc and manganese movement in developing wheat grains. Australian Journal of Plant Physiology, v.25, p.139-144, 1998.

PERVEEN, S. Comparison of Zn extractable bioavailable methods and its critical level in soil and plant. Sarhad Journal of Agriculture, v.16, p.305-317, 2000.

RENGEL, Z. Physiological mechanisms underlying differential nutrient efficiency of crop genotypes. In: RENGEL, Z. (Ed.) Mineral nutrition of crops: fundamental mechanisms and implications. Binghamton: Food Products Press, 1999. p.227-265.

SAFAYA, N.M.; GUPTA, A.P. Differential susceptibility of corn cultivars to zinc deficiency. Agronomy Journal, v.71, p.132-136, 1979.

SHUKLA, V.C.; RAJ, H. Relative response of corn, pearl-millet, sorghum and cowpea to zinc deficiency in soil. Journal of Plant Nutrition, v.10, p.2057-2067, 1987.

SIDDIQI, M.Y.; GLASS, A.D.M. Utilization index: A modified approach to estimation and comparison of nutrient utilization efficiency in plants. Journal of Plant Nutrition, v.4, p.289-302, 1981.

TREHAN, S.P.; SHARMA, R.C. Phosphorus and zinc uptake efficiency of potato (Solanum tuberosum) in comparison to wheat (Triticum aestivum), maize (Zea mays) and sunflower (Helianthus annuus). Indian Journal of Agricultural Sciences, v.70, p.840-845, 2000.

WELCH, R.M. Importance of seed mineral nutrient reserves in crop growth and development. In: RENGEL, Z. (Ed.) Mineral nutrition of crops: fundamental mechanisms and implications. Binghamton: Food Products Press, 1999. p.205-226.

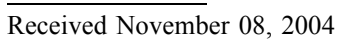

Accepted February 04, 2005 